\title{
Basiskonzepte im GW-Unterricht: Beispiel Märkte, Regulierung und Deregulierung
}

\author{
stefan.hinsch@univie.ac.at, Abendgymnasium Wien \& Universität Wien
}

eingereicht am: 13.03.2017, akzeptiert am: 20.06.2017

\begin{abstract}
Die im August 2016 durch das Unterrichtsministerium veröffentlichte neue Version des Lehrplans für die AHS Sekundarstufe II enthält Basiskonzepte der Geographie und Ökonomie. Basiskonzepte dienen der Erarbeitung wesentlicher Strukturen geographischen und ökonomischen Denkens und orientieren den Unterricht in Richtung eines transferierbaren Wissens. Basiskonzepte können damit den Schlüssel zu den Anforderungsbereichen II (Transfer) und III (Reflexion) darstellen. In diesem Artikel soll eine mögliche Anwendung im Unterricht anhand des Basiskonzeptes Märkte, Regulierung und Deregulierung dargestellt werden.
\end{abstract}

Keywords: Basiskonzepte; Lehrplan Geographie und Wirtschaftskunde; Märkłe, Regulierung und Deregulierung

\section{Key concepts in geography and economics education: The example of markets, regulation and deregulation}

The New Curricula for the Allgemeinbildende Höhere Schulen, which was published in August of 2016, contains core concepts of geography and economy. Core concepts aim to achieve the construction of central structures of geographical and economical thinking. They orient classwork towards transferable knowledge. They can be key for the competencies of transfer and reflection. This article proposes classroom applications for the core concept of markets, regulation and deregulation.

Keywords: key concepts; geography and economics curriculum; market; regulation; deregulation

\section{Basiskonzepte im neuen Lehrplan}

Basiskonzepte versuchen Grundstrukturen und wesentliche Konzepte des ökonomischen und geographischen Denkens exemplarisch zu erarbeiten. Sie dienen der Strukturierung von Inhalten und fokussieren Unterricht in Richtung eines transferierbaren und weniger leicht veraltenden Konzeptwissens. (vgl. Sander 2010; Ketterlin-Geller \& McCoy 2004)

Im neuen Lehrplan der AHS-Oberstufe sind insgesamt 13 Basiskonzepte formuliert. Sie werden aus geographischen, allgemein sozialwissenschaftlichen und ökonomischen Zugängen entwickelt. Am stärksten in Debatten der Ökonomie verwurzelt sind dabei neben Märkte, Regulierung und Deregulierung auch Arbeit, Produktion und Konsum sowie Wachstum und Krise (Bundesministerium für Bildung 2017). Im Allgemeinen ist jedoch davon auszugehen, dass eine Debatte über Konzeptwissen mit dem Neuen Lehrplan der Sekundarstufe II gerade für Österreich erst eröffnet wurde und in der Zukunft Basiskonzepte ersetzt oder anders verstanden werden. Die entscheidende Herausforderung für den GW-Unterricht der nächsten Jahre ist dabei ein grundsätzlicher Übergang zu einem auf Konzeptwissen basierenden Unterricht, nicht ein sklavisches Festhalten an Lehrplanformulierungen.

\section{Anbindung an die ökonomische Debatte}

Die im Lehrplan gesetzten Basiskonzepte sind dabei multiperspektivisch, sie lassen unterschiedliche $\mathrm{Zu}$ gänge zu und erfordern diese auch. Für die Volkswirtschaftslehre ist das interessanterweise nicht selbstverständlich. Die dominante Strömung der letzten Jahrzehnte war die Neoklassik (mehr oder weniger abgeschlossen, mehr oder weniger offen für andere Einflüsse). Bis heute sind viele universitäre Curricula von neoklassischer Modellbildung geprägt (vgl. Earl et al. 2016: $35 \mathrm{ff}$.). 
In der Welt nach der Krise von 2008 ist es nicht mehr möglich, ökonomisches Denken so stark von einer einzigen Strömung der Volkswirtschaftslehre bestimmen zu lassen, zu offensichtlich war die Fehleinschätzung der Situation der Weltwirtschaft. In der Folge werden in der Lehrplanfassung des Basiskonzepts Märkte, Regulierung und Deregulierung Märkte institutionell und staatlich eingebettet beschrieben (Bundesministerium für Bildung 2017) und das Basiskonzept Arbeit, Produktion und Konsum zur Seite gestellt. Denn für die Neoklassik sind Produktion und Konsum nicht vom Konzept der Märkte zu trennen, sie beginnt ihre Überlegungen mit Bedürfnissen (man spricht von consumer sovereignity) und bewegt sich von dort zum Markt, der Bedürfnisse und Produktion als Angebots- und Nachfragefunktion integriert (vgl. Goodwin et al. 2009: 250 f., 490 f.). Ökonomie wird in der Neoklassik zur "Wissenschaft von Märkten“ Peter Bofinger wählt diesen Untertitel für sein Lehrbuch (vgl. Bofinger 2011).

Diese Vorgehensweise ist aber nicht naturgegeben. Exemplarisch herausgegriffen sei die Klassik (besonders David Ricardo) sowie mit dieser verwandte marxistische Ansätze, die mit Produktion und Wert beginnen und von dort die gesellschaftlichen Verhältnisse der Produktion entwickeln (vgl. Sowell 2006: 48 ff.). Oder ein Schumpeter'scher Ansatz, in dem ebenso die Produktion den Ausgangspunkt darstellt, um dort nach den Bedingungen für Innovationen zu fragen (vgl. Mazzucato 2013). Oder Ansätze der Konsumökonomie, die die Reduktion von Konsum und Verbraucher/innen auf eine Nachfragekurve ablehnen (vgl. Fridrich et al. 2017).

Auch die innerhalb und außerhalb der Neoklassik vertretenen Vorstellungen von Märkten sind divers. Es können Ansätze unterschieden werden, die das Gleichgewicht in den Vordergrund rücken (vgl. Debreu 1959) oder Marktversagen untersuchen (vgl. Pigou 1932); Jene, die rationale Individuen und effiziente Märkte betonen (vgl. Fama 1970) und solche, die Instabilitäten erklären wollen (vgl. Stiglitz 2012).

Im Rahmen dieses Artikels werden einige dieser Debatten aufgegriffen (ein erschöpfendes Nachzeichen ist unmöglich), aber es seien vor allem zwei Dinge betont: Einmal erfordert und ermöglicht das Basiskonzept Märkte unterschiedliche Perspektiven und Theorien. Zum Zweiten ist der Kern des Basiskonzepts die Koordinierung von Wirtschaft in einem Spannungsfeld zwischen dezentraler Preisbildung und staatlicher Regulierung und Deregulierung. Es fasst nicht im weiteren Sinn alles, was irgendwie mit Wirtschaft zu tun hat.

Im Folgenden wird versucht für das Basiskonzept Märkte, Regulierung und Deregulierung eine mögliche Umsetzung im GW-Unterricht zu zeigen. Um den
Artikel auch für Praktiker/innen gut zugänglich zu halten, wird versucht eine Besprechung unterschiedlicher Zugänge zu Märkten unmittelbar mit Vorschlägen für die Unterrichtsarbeit zu verbinden. Es folgt daher ein kleiner Durchgang durch eine Reihe von Unterrichtsbeispielen. Der Schwerpunkt liegt auf der Sekundarstufe II, für deren Lehrplan die Basiskonzepte entwickelt wurden. Aber Basiskonzepte und auch das Basiskonzept des Marktes, funktionieren in der Sekundarstufe I gleichermaßen.

\section{Geschäftslokale und Mietwohnungen}

Es ist zu hoffen, dass das erste Beispiel nicht zu sehr verwirrt, denn im Lehrplan der 5. Klasse fallen Lehrplaninhalt (Markt) und Basiskonzept (Markt) weitgehend zusammen. Hier hilft das Basiskonzept dabei, den Blick auf das Wesentliche zu schärfen.

In Schulbüchern findet sich in der Regel ein CrashKurs in neoklassischer Modellbildung (vgl. Wohlschlägel et al.: $54 \mathrm{ff}$.), gefolgt von graphischer Repräsentation des doch eher abstrakten Marktmodells und etwas verkrampfter Schüler/innenorientierung, etwa Kirschen auf Marktständen (ebd.: 59). Zu hohe Abstraktion des Modells und zu hohe Banalität der Beispiele verbauen dabei manchmal Möglichkeiten selbstständigen und entdeckenden Lernens, während manche Detailinformationen wirkliches Spezialwissen darstellen etwa Ausführungen zu Grenznutzen, dem ,Gesetz der Nachfrage' und der Einkommenselastizität (ebd.: 54).

Ein alternativer Vorschlag wäre das Suchen von Geschäftslokalen zur Miete auf Immobilienplattformen (in Gruppenarbeit) und der Vergleich von Quadratmeterpreisen der unterschiedlichen Lagen. Mit entsprechenden Fragestellungen können in der Folge Ursachen für die Preisunterschiede gesucht werden. (Dafür ist es wichtig, dass die Standorte halbwegs bekannt sind, die abgefragte Region aber groß genug für einen halbwegs liquiden Markt ist.)

Eine solche Vorgehensweise veranschaulicht die dezentrale Koordination von Akteurinnen und Akteuren durch Markt und Marktpreis, den eigentlichen Kern des Konzeptes. Gleichzeitig wird die Auseinandersetzung mit vorhandenen Präkonzepten erlaubt. So gehen Schülerinnen und Schüler vielfach davon aus, dass Preise innerhalb bestimmter durch den Staat vorgegebener Richtwerte gesetzt werden (was in vielen Fällen auch zutrifft). Abstrakte Kirschenmärkte laufen Gefahr, zu wenig anschlussfähig zu sein und lassen in der Folge vorhandene Präkonzepte parallel bestehen. Aber genau das muss vermieden werden, Lernen nach Konzepten sollte Präkonzepte aufgreifen und mit ihnen in Dialog treten, um eine Weiterentwicklung zu ermöglichen (vgl. Besand et al. 2011: 167). 
Der Immobilienmarkt ermöglicht auch einen raschen Perspektivenwechsel: Neoklassische Märkte sind das Aufeinandertreffen von Angebot und Nachfrage, aber Märkte sind auch soziale Organisationen, mit dem Ziel Austausch zu ermöglichen. Als solche unterliegen die Regeln des Austauschs Traditionen und administrativen Entscheidungen und sind einem historischen Wandel unterworfen (vgl. Goodwin et al. 2009: 42 ff.; 61 ff.). Im Fall des Immobilienmarktes gelangt man zu Internetplattformen, Maklerinnen und Maklern und spezialisierten Anwältinnen und Anwälten; es findet sich ein Set an Traditionen, Verhaltensweisen und informellen Regeln sowie natürlich rechtlich-administrative Rahmenbedingungen. Der Staat (aber auch andere Organisationen), der zuvor bei Koordination und Preisbildung nicht vorgekommen ist, kommt nun als notwendige Instanz der Regulierung, als Schaffer von Märkten, wieder zurück. Dieser Punkt ist ein wichtiger: Ein neoklassisches Konzept von Markt und Marktmacht ermöglicht vieles, verschließt anderes. Es ist kaum in der Lage Märkte als Organisationen zu fassen; Staat, Gesellschaft und Politik sind dem Modell extern (vgl. ebd.: 74).

Wenn man das rudimentäre Unterrichtsbeispiel nur leicht modifiziert, kann es in anderen Teilen des Lehrplans genauso verwendet werden: Werden Geschäftslokale durch Mietwohnungen ersetzt, wird sozialräumliche Segregation durch den Immobilienmarkt greifbar - mit einem Konzept des Marktes lassen sich also Teile der Stadtgeographie (8. Klasse) fassen. Die Auswahl der Inhalte wird dadurch gestrafft, und den Schülerinnen und Schülern eröffnen sich Möglichkeiten der Selbstständigkeit; denn wenn ein Konzept dezentraler Koordination und Preisbildung durch einen Markt vorhanden ist, kann es auch selbsttätig auf andere Phänomene (wie die sozialräumliche Segregation) angewandt werden.

Der Markt für Mietwohnungen macht auch problematische Marktergebnisse greifbar, denn der Markt für Mietwohnungen ist voller Externalitäten - und daher in Österreich durch umfangreiche staatliche Regulierung eingeschränkt. Dahinter steht das Konzept des Marktversagens des Neoklassikers Pigou (1932: $14 \mathrm{ff}$.). Ein freier Wohnungsmarkt ist unzweifelhaft sehr effizient darin, Leuten mit viel Geld schöne Wohnungen zu verschaffen, und Leuten mit wenig Geld schlechtere - aber die Problematik sozialräumlicher Segregation und Ghettobildung muss für ein Publikum aus Geographinnen und Geographen nicht weiter behandelt werden.

Der Wohnungsmarkt eignet sich auch für ein selbsttätiges und entdeckendes Lernen staatlicher Regulierung, da Fragen des Mietrechts über Artikel, Exkursionen (Mietervereinigung, Arbeiterkammer) oder Expert/innengespräche gut zugänglich sind. Auch wird die Interessensleitung und Konflikthaftigkeit staatlicher Regulierung greifbar, denn Ausmaß und Zielrichtung der staatlichen Eingriffe sind ja Resultat einer politischen Auseinandersetzung, letztlich einer Auseinandersetzung um Marktmacht. Auch das wird deutlich, wenn man unterschiedliche Positionen unterschiedlicher Organisationen zu aktuellen Fragen der Veränderung des Mietrechtes (etwa Arbeiterkammer und Wirtschaftskammer) gegenüberstellt. Und schließlich betrifft der Wohnungsmarkt nicht nur die Gesellschaftsökonomie, sondern auch individuelle Fragen der Konsumökonomie - etwa ob die Altbaumiete illegal zu hoch ist.

Damit ermöglicht eine Untersuchung sozialräumlicher Segregation über den Markt für Mietwohnungen die Beschäftigung mit dem Kern des Basiskonzeptes: das Spannungsfeld zwischen dezentraler Koordinierungund staatlicher Regulierung (oder Deregulierung).

\section{Marktmacht und asymmetrische Informationen}

Das Vorliegen von Marktmacht verändert ein Marktergebnis. Man spricht von oligopolistischen oder monopolistischen Märkten (vgl. Bofinger 2011: 115ff.). Jedes Mal, wenn Unterrichtsthemen Märkte beinhalten, ist eine kurze Analyse der Marktstruktur möglich - der Eintrittsbarrieren, der Form der Konkurrenz, der Marktmacht - um damit Rückschlüsse auf die Verteilung von Macht und Einkommen zwischen den Marktteilnehmerinnen und -teilnehmern ziehen zu können.

Es gibt etwa zahlreiche Unterrichtsbeispiele über Fair Trade und Globalisierung, die Ungerechtigkeit thematisieren (der/die Näher/in erhält nur $0,4 \%$ des Preises eines Turnschuhs, Ausbeutung in der Fertigung von Mobiltelefonen, Kakaobauern und -bäuerinnen bekommen nur etwa $5 \%$ des Preises der Schokolade, usw.). Fair und unfair werden üblicherweise am moralischen Empfinden eines gerechten Preises festgemacht, etwa der Frage, welchen Anteil des Schokoladepreises die Schülerinnen und Schüler den Kakaoproduzentinnen und -produzenten zusprechen würden. (vgl. OroVerde 2011). Ein solcher fairer Preis wird in der Wirtschaftsethik aufgegriffen (vgl. Hecker 2008) und blickt zurück auf eine Traditionslinie von Aristoteles bis Thomas von Aquin (vgl. ebd.: $44 \mathrm{ff}$.). Aber ein stärker im Mainstream verankertes Konzept von Markt und Marktmacht hilft dabei, weitere Perspektiven zu gewinnen.

Denn Marktmacht ist entlang der Wertschöpfungskette nicht gleich verteilt: Die Schokoladehersteller und die Einzelhandelsketten haben viel Marktmacht, die Handelshäuser haben Marktmacht, aber die einzelnen Kakaobauern und -bäuerinnen haben 
praktisch keine Marktmacht und ihre Kindersklaven haben noch weniger. Der Vorstandsvorsitzende von Nestlé beispielsweise verdient sehr gut, weil er in einem Kreis anderer Vorstandsvorsitzender sein Gehalt mehr oder weniger selbst festlegt (sehr viel Marktmacht), die Kakaobauern und -bäuerinnen befinden sich lediglich in Abhängigkeit von ihrem Lohn und sind gezwungen, die Arbeitskraft und die Produkte zu einem sehr geringen Preis zu verkaufen. Es ist auch in der Sekundarstufe I einfach vermittelbar, dass Nestlé einen Verhandlungsvorteil gegenüber einer Kakaobäuerin oder einem Arbeiter auf der Plantage hat; in der Sekundarstufe II kann man die Marktstruktur weiter analysieren und herausarbeiten, warum es weltweit 5,5 Millionen Kakaokleinbauern und -bäuerinnen gibt (die in der Folge Preise nicht beeinflussen können) (vgl. TAZ 2013), aber nur wenige große Lebensmittelkonzerne: Das liegt an vorhandenen oder nicht vorhandenen Skalenerträgen (Vorteilen der Massenproduktion), die in zahlreichen Märkten eine große Zahl von Anbietern unmöglich machen, weil die Kosten bei kleinen Stückzahlen zu hoch sind.

Das Konzept des gerechteren Preises bringt als Antwort auf die obigen Problemstellungen (arme Kakaobauern und -bäuerinnen und Näher/innen) den ethischen Konsum (z. B. Fair-Trade-Produkte und Clean Clothes). Das ist als erster Schritt sinnvoll und ermöglicht eine unmittelbare Handlung der Schüler/innen. Ein Konzept von Marktmacht ergänzt eine andere Perspektive auf mögliche Lösungen. Etwa Ansätze, die Marktmacht erhöhen, wie Gewerkschaften oder Produzentenkartelle. Oder Ansätze der Industriepolitik, mit der die betroffenen Länder versuchen können aus armutserzeugender Produktion auszusteigen.

Tatsächlich können sich - auch im Unterricht die verwendeten Konzepte gegenseitig hinterfragen, sie werden dadurch lebendiger und flüssiger. Ein Konzept der Marktmacht kann die Beschränkungen des ethischen Konsums aufzeigen, der ein Empowerment der Produzenten eben nur bedingt ermöglicht, denn die Zentrale für Fair Trade International steht in Bonn, nicht in Abidjan oder Accra, und die Handelnden sind europäische Konsumentinnen und Konsumenten, keine afrikanischen Bäuerinnen und Bauern. Das Konzept eines ,gerechten Preises' kann seinerseits das neoklassische Marktmodell selbst hinterfragen: Dieses geht immer von Nutzenmaximierer/innen aus, die eigentlich so wenig wie möglich bezahlen möchten - plötzlich sieht man die Bereitschaft zahlreicher Menschen wirtschaftliche Entscheidungen nach anderen Kriterien zu treffen.

Dies war lediglich ein Beispiel: Es gibt eine große Menge von Lehrplanthemen, bei denen ein Konzept der Marktmacht Inhalte neu strukturieren kann. Eine unvollständige Liste: die Problematik natürlicher
Monopole (und damit verbundener Marktmacht), etwa in Verbindung mit der Privatisierung öffentlicher Dienstleistungen oder der Wasserversorgung; die Bedeutung von Marktmacht von Unternehmen und Gewerkschaften auf dem Arbeitsmarkt für die Lohnhöhen; Veränderungen von Marktmacht (zwischen Unternehmen und Gewerkschaften) im Zuge von Globalisierung, Freihandel, EU-Binnenmarkt und einer heute möglichen Drohung mit Produktionsauslagerung. In Betriebswirtschaftslehre und Marketing der Versuch von Unternehmen Marktmacht zu erhalten, Märkte zu dominieren, oder sich über Alleinstellungsmerkmale von der Konkurrenz abzugrenzen - für den Unterricht lohnend etwa die Werbestrategie von Red Bull oder die Analyse einer Autowerbung.

Werbebeispiele erlauben weitere Multiperspektivität: Sie gewähren einen Zugang zur Produktion von Bedürfnissen (was eher dem Basiskonzept Arbeit, Produktion und Konsum zuzuordnen wäre), sie ermöglichen eine Auseinandersetzung mit Marktmacht (neoklassische Perspektive), aber sie zeigen auch konkreten Wettbewerb. Während in der Neoklassik Wettbewerb ein mathematisierbarer Zustand eines Marktes ist, bedeutet Wettbewerb etwa für die österreichische Schule (und im Alltagsgebrauch des Begriffs) einen Prozess, in dem unterschiedliche Unternehmen tatsächlich miteinander wetteifern (vgl. Hayek 1968). Werbebeispiele machen auch erlebbar, dass manche Märkte von Unternehmen geschaffen werden - etwa der Markt für Energydrinks.

So wie die Marktmacht gehört auch das Vorhandensein von Informationsasymmetrien - bei denen einige Marktteilnehmer/innen mehr wissen als andere - zu den in der ökonomischen Wissenschaft allgemein anerkannten Gründen von Marktversagen. Denn das neoklassische Standardmodell postuliert vollständige Informationen aller Marktteilnehmer/innen (vgl. Stiglitz 2000). Im Unterricht ergeben sich hier Anwendungen für die Konsumökonomie. Das dahinterliegende Konzept bedeutet: Vorsicht bei intransparenten Märkten (wenn die Verkäuferin mehr über das Produkt weiß als ich). Und: Habe ich wirklich verstanden, was ich da kaufe? Eine Untersuchung des Handyvertrages oder des Jugendkontos auf versteckte Kosten ist ein Beispiel. Fächerübergreifend mit Biologie und im Lehrplanbereich der Wettbewerbspolitik kann die Lebensmittelampel besprochen werden, die durch eine rot-gelb-grün Kennzeichnung für Transparenz in Bezug auf Nährwert bzw. für eine Einteilung in gesund/ungesund sorgen sollte, bisher aber am Widerstand der Lebensmittelindustrie scheitert. Diese kann auf Lieblingsspeisen oder allgemein bekannte Produkte angewendet werden. Wobei die Biologie die Nährwertanalyse übernimmt, der GW-Unterricht die politische Auseinandersetzung in der Wettbewerbspo- 
litik - den Konflikt um Regulierung und Deregulierung.

Vollständig symmetrische Informationen (vollständige Transparenz) sind in den allermeisten Fällen unmöglich: Wer einer Bank Geld borgt, kann Konditionen für Jugendkonten vergleichen, wird aber über Risiken in der Bankbilanz (die eine Pleite auslösen könnten) weniger wissen als die Vorstandsmitglieder der Bank. Und wer Kartoffelchips kauft, wird mit oder ohne Lebensmittelampel weniger über die Inhaltsstoffe wissen als der Herstellerfirma oder ein/e Lebensmitteltechniker/in. Aber letzten Endes ist es eine Frage von politischen Prozessen und Macht, wie viel versteckt werden darf: Hier lassen sich im Unterricht nicht nur die Auseinandersetzungen um die Lebensmittelampel nachzeichnen, sondern auch die bereits erwähnten Konflikte um das österreichische Mietrecht. Bei diesen geht es nicht nur um die legal zulässige Höhe von Altbaumieten, sondern auch um die Transparenz des Mietpreises.

\section{Jenseits der Neoklassik: Dynamik und Instabilität}

Natürlich gibt es Konzepte vom Markt, die weit von der Neoklassik entfernt sind. Sie sind überall dort besonders sinnvoll aufzugreifen, wo es um dynamische Marktprozesse, Innovationen, Veränderung und Krisenerscheinungen geht. Etwa postkeynesianische Ansätze (vgl. Minsky 1986; Koo 2015) oder Ansätze der Verhaltensökonomie (vgl. Akerlof \& Shiller 2009) um die Instabilität von Finanzmärkten zu greifen. Oder Überlegungen radikaler Unsicherheit (häufig in einer Tradition der österreichischen Schule der Nationalökonomie), die ebenfalls Krisenphänomene fassen können (vgl. King 2016) oder Wachstum und Innovationen zu erklären versuchen (vgl. Mazzucato 2013). Denn ein neoklassisches Marktmodell dient in erster Linie der Erklärung von Gleichgewichtszuständen. Der Kern der Neoklassik - die allgemeine Gleichgewichtstheorie postuliert eine Auktion vor dem Beginn der Zeit, in der alle Wirtschaftsteilnehmer/innen für sie optimale (Grenzkosten ist Grenznutzen) relative Preise bekannt geben, die wiederum allen anderen bekannt sind. Und diese relativen Preise gelten für aktuelle Märkte und für alle möglichen zukünftigen.

Für den Unterricht spannend ist zum Beispiel die Untersuchung von Immobilienblasen und daraus folgender finanzieller Instabilität. Bewährt hat sich dabei eine Mischung aus anekdotenhaften Zeitungsartikeln (etwa über die Errichtung von Geisterstädten in China oder Andalusien) und einer verbindenden Analyse von Immobilien-Preis-Indices und privater Haushaltsverschuldung, gemessen an den verfüg- baren Einkommen oder dem BIP (Daten sind über die OECD mittlerweile leicht zugänglich). Das kann dann durch die Brille einer postkeynesianischen Hypothese finanzieller Instabilität betrachtet werden: Um zukünftige Entwicklungen abschätzen zu können, schreiben Menschen die jüngere Vergangenheit einfach fort. Steigende Preise lösen die Erwartung weiter steigender Preise aus und können so zu einer Zunahme von Risiko und Schuldenpositionen im Finanzsystem führen. (vgl. Pettis 2013: 69 ff.)

Ein Unterrichten mit Basiskonzepten bedeutet in diesem Fall nicht einfach: Finanzkrisen sind auch wichtig. Es geht darum Material so auszuwählen, dass man in einer anekdotenhaften Beschreibung von Finanzkrisen und der Aufzählung von Verwerfungen nicht verlorengeht (so wichtig die Anekdoten und die spannenden Geschichten auch sind). Letzten Endes sollen die Schüler/innen ein Konzept über die Instabilität von Finanzmärkten entwickeln können. So ein Konzept wird dann zu einem übertragbaren Erklärungsinstrument, welches selbsttätig auf neue Phänomene angewandt werden kann.

\section{Probleme und das Anforderungsniveau II und III}

Die Liste an Inhalten, die mit Konzepten und Theorien des Marktes strukturiert werden können, ist nicht erschöpft. Im Prinzip lässt sich über jedes wirtschaftliche Thema ein Bezug auf Markt und Marktstruktur herstellen. Bei jeder Frage der Wirtschafts- oder Regionalpolitik kann man analysieren, warum eine dezentrale Koordination über Marktpreise möglicherweise unzureichend ist, welches spezifische Problem der Marktstruktur einen gesellschaftlich-politischen Eingriff notwendig oder wünschenswert macht. Und bei jeder dieser Fragen lässt sich auch ein Konflikt über die Art und Intensität dieses gesellschaftlich-politischen Eingriffs fassen.

Aber selbstverständlich wird es nicht in jeder Situation angebracht erscheinen, Unterricht auf ein Konzept des Marktes auszurichten. Und um Konzepte und Teilkonzepte einsetzen und hinterfragen zu können, müssen sie durch die Lehrperson ausreichend verstanden sein. Bei einer Liste von 13 Basiskonzepten - und bei hier im Artikel gestreiften Theorien wie der Hypothese finanzieller Instabilität - kann das nicht durchgehend erwartet werden. Das macht nichts, denn ein Lehrplaninhalt lässt sich von verschiedenen Seiten erschließen.

Basiskonzepte bieten dabei einen einfachen Schlüssel zum Anforderungsbereich II und III. Wissen kann nicht selbstständig angewandt oder transferiert werden, wenn es nicht zuvor als transferierbares Wissen, 
als Konzeptwissen, vorliegt. Das Mitdenken von Basiskonzepten im Unterricht bedeutet das Mitdenken von Transfermöglichkeiten. Mit dem Basiskonzept Markt wird aus einer Liste der Probleme des Tourismus (an die Tafel geschrieben und gelernt) eine Reflexion und Begründung der oft harten und schlecht bezahlten Arbeit im Tourismussektor. Denn wenn es einmal gelingt die Marktstruktur zu erfassen, können die Schüler/innen aus dem Wissen über geringe Eintrittsbarrieren eine intensive Konkurrenz zwischen Regionen, Betrieben und Beschäftigten und schließlich Druck auf Preise, Margen, Löhne und Einkommen ableiten. Und statt einer Erzählung über Alternativen zum Massentourismus, können diese auf ihre Wirksamkeit hinsichtlich der Veränderung von Marktstrukturen beurteilt werden.

Ein Unterricht, der verstärkt auf Konzepte setzt, führt keine neuen Lehrplaninhalte oder Unterrichtsthemen ein. Aber er führt weg von aufzählendem und erzählendem hin zu transferierbarem Wissen.

\section{$7 \quad$ Literatur}

Akerlof, G., A. \& R. J. Shiller (2009): Animal Spirits. How Human Psychology Drives the Economy, and Why It Matters for Global Capitalism. Princeton und Oxford: Princeton University Press.

Besand, A., T. Grammes, R. Hedtke, P. Henkenborg, D. Lange, A. Petrik, S. Reinhardt \& W. Sander (2011): Sozialwissenschaftliche Basiskonzepte als Leitideen der politischen Bildung - Perspektiven für Wissenschaft und Praxis. In: Autorengruppe Fachdidaktik: Konzepte der politischen Bildung. Eine Streitschrift. Schriftenreihe der Bundeszentrale für politische Bildung 1141/2011. S. 163-171.

Bofinger P. (2011): Grundzüge der Volkswirtschaftslehre. Eine Einführung in die Wissenschaft von Märkten. Halbergmoos: Pearson.

Bundesministerium für Bildung (2017): Gesamte Rechtsvorschrift für Lehrpläne - allgemeinbildende höhere Schulen, Fassung vom 1.9.2017. https://www.ris.bka. gv.at/GeltendeFassung.wxe?Abfrage=Bundesnormen\&G esetzesnummer $=10008568 \&$ Fassung Vom $=2017-09-01$ (15.6.2017).

Earl, J., C. Moran \& Z. Ward-Perkins (2016): The Econocracy. The Perils of Leaving Economics to the Experts. Manchester: Manchester University Press.

Debreu, G. (1959): Theory of Value. An Axiomatic Analysis of Economic Equilibrium. New Haven und London: Yale University Press. http://cowles.yale.edu/sites/ default/files/files/pub/mon/m17-all.pdf (3.2.2017).

Fama, E. F. (1970): Efficient Capital Markets. A Review of Theory an Empirical Work. In: Journal of Finance 25/2. S. 383-417. www.e-m-h.org/Fama70.pdf (28.2.2017)

Fridrich, C., R. Hübner, K. Kollmann, M. Piorkowski \& N. Tröger (2017): Abschied vom eindimensionalen Verbraucher. Wiesbaden: Springer.

Goodwin, N., J. A. Nelson \& J. Harris (2009): Macroeconomics in Context. Armonk und London: M.E. Sharpe.
Hayek, F. A. (1968): Der Wettbewerb als Entdeckungsverfahren. In: Internationales Institut ,Österreichische Schule der Nationalökonomie" (Hrsg.): Die Österreichische Schule der Nationalökonomie. Texte - Band II von Hayek bis White. Wien: Manz'sche Verlagsund Universitätsbuchhandlung. S. 119-137. https:// mises.org/library/competition-discovery-procedure-0 (28.2.2017).

Hecker, C. (2008): Lohn- und Preisgerechtigkeit. Marburg: Metropolis.

King, M. (2016): The End of Alchemy. Money, Banking and the Future of the Global Economy. London: Little, Brown.

Ketterlin-Geller, L., R. und J. D. McCoy (2004): Rethinking Instructional Delivery for Diverse Student Populations. Serving All Learners with Concept Based Instruction. In: Intervention in School and Clinic, 40/2. S. 88-95. http://www.whatisib.com/concept-based-learning.html (3.3.2017).

Koo, R. C. (2015): The Escape from Balance Sheet Recession and the QU Trap. A Hazardous Road for the World Economy. Singapur: Wiley.

Mazzucato, M. (2013): The Entrepreneurial State. Debunking Public vs. Private Sector Myths. London und New York: Anthem.

Minsky, H. P. (2008/1986): Stabilizing an Unstable Economy. New York: McGraw-Hill.

OroVerde Tropenwaldstiftung (2011): Kakao. http://regenwald-unterrichtsmaterial.oroverde.de/unterrichtseinheiten/kakao/1-einfuehrung/ (28.2.2017).

Pettis, M. (2013): The Great Rebalancing. Trade, Conflict, and the Perilious Road Ahead for the World Economy. Princeton und Oxford: Princeton University Press.

Pigou, A. C. (1932): The Economics of Welfare. London: Macmillan. Onlineverfügbar unter: http://oll.libertyfund.org/titles/1410 (28.2.2017).

Sander, W. (2010): Wissen im kompetenzorientierten Unterricht - Konzepte, Basiskonzepte, Kontroversen in den gesellschaftswissenschaftlichen Fächern. In: Zeitschrift für Didaktik der Gesellschaftswissenschaften 1/1. S. 42-67.

Sowell, T. (2006): On Classical Economics. Yale: Yale University Press.

Stiglitz, J. (2000): The Contributions of the Economy of Information to Twentieth Century Economics. The Quarterly Journal of Economics 115/4. S. 1441-1478. ricardo.ecn.wfu.edu/ - cottrell/papers/stiglitz.pdf (28.2.2017).

Stiglitz, J. (2012): The Price of Inequality. How Today's Divides Society Endangers Our Future. New York und London: Norton.

TAZ (2013): Bittere Zeiten für Kakaobauern. http:// www.taz.de/Herstellung-von-Schokolade/!5070608/ (28.2.2017)

Wohlschlägel, H. et al.(2013): Durchblick 5. Wien: Westermann. 